\title{
First-order optical systems with unimodular eigenvalues
}

\author{
Martin J. Bastiaans \\ Faculteit Elektrotechniek, Technische Universiteit Eindhoven, Postbus 513, 5600 MB Eindhoven, Netherlands
}

Tatiana Alieva

Facultad de Ciencias Físicas, Universidad Complutense de Madrid, Ciudad Universitaria s/n, Madrid 28040, Spain

Received November 10, 2005; revised February 7, 2006; accepted February 10, 2006; posted February 17, 2006 (Doc. ID 65869)

It is shown that a lossless first-order optical system whose real symplectic ray transformation matrix can be diagonalized and has only unimodular eigenvalues is similar to a separable fractional Fourier transformer in the sense that the ray transformation matrices of the unimodular system and the separable fractional Fourier transformer are related by means of a similarity transformation. Moreover, it is shown that the system that performs this similarity transformation is itself a lossless first-order optical system. Based on the fact that Hermite-Gauss functions are the eigenfunctions of a fractional Fourier transformer, the eigenfunctions of a unimodular first-order optical system can be formulated and belong to the recently introduced class of orthonormal Hermite-Gaussian-type modes. Two decompositions of a unimodular first-order optical system are considered, and one of them is used to derive an easy optical realization in more detail. (C) 2006 Optical Society of America

OCIS codes: $070.2580,070.4690,080.2730,120.4820$.

\section{INTRODUCTION}

Recently there has been some interest in signal transformations that possess unimodular eigenvalues, especially in view of the easy fractionalization of such transformations. ${ }^{1,2}$ In particular, we mention cyclic transformations, ${ }^{3-5}$ for which the eigenvalues are not only $2 \pi$ periodic, but, in addition, the argument of each eigenvalue shows a rational relationship to $2 \pi$.

One of the important transformations used in optical signal processing is the linear canonical integral transformation, ${ }^{6,7}$ which corresponds to a first-order optical system. ${ }^{8}$ In this paper we consider lossless first-order optical systems that have the additional property that the eigenvalues of their real symplectic ray transformation matrices ${ }^{8,9}$ are unimodular; moreover, we require that the ray transformation matrix can be diagonalized. Such systems and ray transformation matrices will be called unimodular; although not explicitly stated, the term unimodular thus implicitly implies that the ray transformation matrix is diagonalizable.

Sections 2 and 3 are devoted to some preparatory work on the description of a general lossless first-order optical system, its real symplectic ray transformation matrix $\mathbf{T}$, and the linear canonical integral transformation that such a system performs on an optical signal. In Section 4, we turn our attention to a system with a diagonalizable ray transformation matrix, and we determine the eigenvalues $\lambda_{n}$ and the eigenvectors $\mathbf{q}_{n}$ of this matrix. In particular, we show that these eigenvalues and eigenvectors can be combined into an eigenvalue matrix $\Lambda$ and an eigenvector matrix $\mathbf{Q}$ in such a way that these matrices are symplectic, albeit complex. This section concludes with the particular forms that the eigenvalue matrix $\boldsymbol{\Lambda}_{f}$ and the eigenvector matrix $\mathbf{Q}_{u}$ take in the special case of a unimodular (and diagonalizable) ray transformation matrix $\mathbf{T}_{u}$. From that point on, we stay in the realm of unimodular systems and ray transformation matrices, and we show in Section 5 that the eigenvector matrix $\mathbf{Q}_{u}$ takes a form that allows us to decompose the unimodular system (with ray transformation matrix $\mathbf{T}_{u}$ ) into a separable fractional Fourier transformer (with ray transformation matrix $\mathbf{T}_{f}$ ) embedded in between a general lossless firstorder subsystem and its inverse (with ray transformation matrices $\mathbf{M}$ and $\mathbf{M}^{-1}$, respectively): $\mathbf{T}_{u}=\mathbf{M} \mathbf{T}_{f} \mathbf{M}^{-1}$. Some examples are treated in Section 6.

In Section 7 we show how this decomposition allows us to formulate the eigenfunctions of the unimodular system. We will conclude that these eigenfunctions belong to the recently introduced class of orthonormal HermiteGaussian-type modes, which arise at the output of a lossless first-order system when the common Hermite-Gauss modes are presented at the system's input. An alternative decomposition, where the Iwasawa decomposition of real symplectic matrices is used, is presented in Section 8 and consists of a so-called orthosymplectic system embedded in between two lenses and two magnifiers. This alternative decomposition has less degrees of freedom than the decomposition $\mathbf{M} \mathbf{T}_{f} \mathbf{M}^{-1}$ (but still enough to reach all possible diagonalizable real symplectic matrices with unimodular eigenvalues) and is thus better suited for the construction of unimodular first-order optical systems. An optical realization based on this alternative decomposition is presented in Section 9.

\section{FIRST-ORDER OPTICAL SYSTEMS}

Any lossless first-order optical system can be described by its ray transformation matrix, ${ }^{8,9}$ which relates the posi- 
tion $\mathbf{r}_{i}$ and direction $\mathbf{p}_{i}$ of an incoming ray to the position $\mathbf{r}_{o}$ and direction $\mathbf{p}_{o}$ of the outgoing ray:

$$
\left[\begin{array}{l}
\mathbf{r}_{o} \\
\mathbf{p}_{o}
\end{array}\right]=\mathbf{T}\left[\begin{array}{l}
\mathbf{r}_{i} \\
\mathbf{p}_{i}
\end{array}\right]=\left[\begin{array}{ll}
\mathbf{A} & \mathbf{B} \\
\mathbf{C} & \mathbf{D}
\end{array}\right]\left[\begin{array}{l}
\mathbf{r}_{i} \\
\mathbf{p}_{i}
\end{array}\right] .
$$

The ray transformation matrix $\mathbf{T}$ of such a system is real and symplectic; symplecticity can be expressed elegantly in the form

$$
\left[\begin{array}{ll}
\mathbf{A} & \mathbf{B} \\
\mathbf{C} & \mathbf{D}
\end{array}\right]^{-1}=\left[\begin{array}{cc}
\mathbf{D}^{t} & -\mathbf{B}^{t} \\
-\mathbf{C}^{t} & \mathbf{A}^{t}
\end{array}\right], \quad \text { or } \mathbf{T}^{-1}=\mathbf{J T}^{t} \mathbf{J}
$$

with

$$
\mathbf{J}=i\left[\begin{array}{cc}
\mathbf{0} & -\mathbf{I} \\
\mathbf{I} & \mathbf{0}
\end{array}\right], \quad \mathbf{J}=\mathbf{J}^{-1}=\mathbf{J}^{\dagger}=-\mathbf{J}^{t},
$$

where $\mathbf{I}$ is the identity matrix and $\mathbf{0}$ is the null matrix; as usual, the superscript $t$ denotes transposition, and the superscript $\dagger$ is used to denote the combined action of transposition and complex conjugation. As some well-known one-dimensional examples we mention

$$
\left[\begin{array}{cc}
1 & \lambda_{\curvearrowright} z \\
0 & 1
\end{array}\right], \quad\left[\begin{array}{cc}
1 & 0 \\
-1 / \lambda_{\circ} f & 1
\end{array}\right], \quad\left[\begin{array}{cc}
0 & w^{2} \\
-w^{-2} & 0
\end{array}\right]
$$

(see Ref. 9 Appendix B.3 and B.4), and in particular, ${ }^{10}$

$$
\mathbf{T}_{f}(\theta)=\left[\begin{array}{cc}
\cos \theta & w^{2} \sin \theta \\
-w^{-2} \sin \theta & \cos \theta
\end{array}\right],
$$

corresponding to a section of free space with distance $z$, a lens with focal length $f$, a Fourier transformer with scaling $w$, and a fractional Fourier transformer ${ }^{10-13}$ with fractional angle $\theta$ (and scaling $w$ ), respectively, acting on light with wavelength $\lambda_{\text {。 }}$.

Two commonly used coherent-optical realizations of (one-dimensional) fractional Fourier transformers ${ }^{10}$ are suggested by Lohmann: setup (a) consisting of one thin (cylindrical) lens with focal length $f$, preceded and followed by two identical distances $z$ of free space, and setup (b) consisting of two identical thin (cylindrical) lenses with focal lengths $f$, separated by a distance $z$. The relation among $z, f$, and the fractional angle $\theta$ reads $z$ $=2 f \sin ^{2}(\theta / 2)$ in both setups, whereas $w^{2}=\lambda_{\text {。 }} f \sin \theta$ in setup (a) and $w^{2}=\lambda_{\circ} f \tan (\theta / 2)$ in setup (b). Usually we work with normalized variables $\mathbf{r}=: \mathbf{r} / w$ and $\mathbf{p}=: \mathbf{p} w$ so that the fractional Fourier transformer [see Eq. (5)] corresponds to a mere rotation in $\mathbf{r p}$ space, $\left(x, p_{x}\right),\left(y, p_{y}\right), \ldots$, through an angle $\theta$. Two crossed one-dimensional fractional Fourier transformers, with different ratios $z / f$, but with identical total length $2 z$ [in $\operatorname{setup~(a)]~or~} z$ [in setup (b)], lead to a two-dimensional, separable fractional Fourier transformer.

The one-dimensional fractional Fourier transformer with ray transformation matrix $\mathbf{T}_{f}(\theta)$ [see Eq. (5)] is a fractional version of the normal Fourier transformer, for which $\theta=\pi / 2$ [see the rightmost matrix in expression (4)]. The ray transformation matrix $\mathbf{T}_{f}(\theta)$ has unimodular eigenvalues $\lambda_{1,2}=\exp ( \pm i \theta)$, and it is well known from the similarity property in matrix theory (Ref. 14, Chap. 13) that when such a matrix is embedded in between a matrix $\mathbf{M}$ and its inverse $\mathbf{M}^{-1}$, the resulting matrix $\mathbf{M T}_{f}(\theta) \mathbf{M}^{-1}$ has the same unimodular eigenvalues. It will be clear that this holds not only in the case of a one-dimensional fractional Fourier transformer, but also in the case of a separable higher-dimensional one, with ray transformation matrix $\mathbf{T}_{f}\left(\theta_{1}, \theta_{2}, \ldots\right)$. Moreover, if $\mathbf{M}$ is a real symplectic matrix (and thus the ray transformation matrix of a lossless first-order optical system), the resulting matrix $\mathbf{M} \mathbf{T}_{f}\left(\theta_{1}, \theta_{2}, \ldots\right) \mathbf{M}^{-1}$ is also real and symplectic.

In this paper we will show that the inverse is also true: Each diagonalizable real symplectic matrix with unimodular eigenvalues is similar to the ray transformation matrix $\mathbf{T}_{f}$ of a separable fractional Fourier transformer and can be written in the form $\mathbf{M} \mathbf{T}_{f} \mathbf{M}^{-1}$, with $\mathbf{M}$ a real symplectic matrix. Note that not all matrices can be diagonalized; the ray transformation matrices that belong to a section of free space and to a lens [see the leftmost and central matrices in expression (4) with their eigenvalues equal to 1] are two obvious optical examples of nondiagonalizable matrices.

\section{LINEAR CANONICAL INTEGRAL TRANSFORMATION}

A lossless first-order optical system performs a linear canonical integral transformation on the signal $f_{i}(\mathbf{r})$ that appears at the system's input:

$$
f_{i}\left(\mathbf{r}_{i}\right) \rightarrow f_{o}\left(\mathbf{r}_{o}\right)=\mathcal{R}\left[f_{i}\left(\mathbf{r}_{i}\right)\right]\left(\mathbf{r}_{o}\right) .
$$

If the system is described by a ray transformation matrix $\mathbf{T}$ whose submatrix $\mathbf{B}$ is nonsingular, the output signal $f_{o}(\mathbf{r})=\mathcal{R}\left[f_{i}\left(\mathbf{r}_{i}\right)\right]\left(\mathbf{r}_{o}\right)$ can be expressed in terms of the Collins integral $^{6,7,15}$ :

$$
\begin{aligned}
f_{o}\left(\mathbf{r}_{o}\right)= & \frac{\exp (i \phi)}{\sqrt{\operatorname{det} i \mathbf{B}}} \int_{-\infty}^{\infty} f_{i}\left(\mathbf{r}_{i}\right) \exp \left[i \pi \left(\mathbf{r}_{i}^{t} \mathbf{B}^{-1} \mathbf{A} \mathbf{r}_{i}-2 \mathbf{r}_{i}^{t} \mathbf{B}^{-1} \mathbf{r}_{o}\right.\right. \\
& \left.\left.+\mathbf{r}_{o}^{t} \mathbf{D B}^{-1} \mathbf{r}_{o}\right)\right] d \mathbf{r}_{i} .
\end{aligned}
$$

The phase factor $\exp (i \phi)$ in Eq. (7) can be chosen rather arbitrarily, for instance to make transformations additive in their determining parameters. As an important onedimensional example we mention the fractional Fourier transformer ${ }^{11-13,16,17}$ with fractional angle $\theta$ (and $0 \leqslant \theta$ $<\pi$ ) for which we have

$$
f_{o}\left(x_{o}\right)=\left\{\begin{array}{l}
\frac{\exp (i \theta / 2)}{w \sqrt{i \sin \theta}} \int_{-\infty}^{\infty} \exp \left[i \pi \frac{\left(x_{o}^{2}+x_{i}^{2}\right) \cos \theta}{w^{2} \sin \theta}\right] \\
\times \exp \left[-i 2 \pi \frac{x_{o} x_{i}}{w^{2} \sin \theta}\right] f_{i}\left(x_{i}\right) d x_{i} \quad(\theta \neq 0), \\
f_{i}\left(x_{o}\right) \quad(\theta=0)
\end{array}\right.
$$

and where we have chosen the phase angle $\phi$ in Eq. (7) such that additivity for the fractional angle $\theta$ holds ${ }^{17}$; note that by properly choosing ${ }^{17}$ the square root of $i \sin \theta$, additivity and $2 \pi$ periodicity can be obtained for $\theta \in \mathbb{R}$. The relation to the ray transformation matrix is clear: $\mathbf{A}=\mathbf{D}$ $=\cos \theta$ and $\mathbf{B} w^{-2}=-\mathbf{C} w^{2}=\sin \theta$, [see Eq. (5)]. We remark that additivity and $2 \pi$ periodicity also hold for the canonical integral transformation with ray transformation matrix $\mathbf{M} \mathbf{T}_{f}(\theta) \mathbf{M}^{-1}$, and that the particular cases $\mathbf{M} \mathbf{T}_{f}(0) \mathbf{M}^{-1}$ 
and $\mathbf{M T}_{f}(\pi) \mathbf{M}^{-1}$ correspond to the transformations $f_{o}(\mathbf{r})$ $=f_{i}(\mathbf{r})$ and $f_{o}(\mathbf{r})=f_{i}(-\mathbf{r})$, respectively.

\section{EIGENVALUES AND EIGENVECTORS OF UNIMODULAR SYSTEMS}

In the present paper we will eventually consider lossless first-order optical systems for which the ray transformation matrix $\mathbf{T}$ has unimodular eigenvalues $\left|\lambda_{n}\right|=1$; moreover, we will restrict ourselves to systems for which $\mathbf{T}$ can be diagonalized. We recall that such systems and their diagonalizable ray transformation matrices $\mathbf{T}_{u}$ will be called unimodular. We will thus get the decomposition

$$
\mathbf{T}_{u}=\mathbf{Q}_{u} \boldsymbol{\Lambda}_{f} \mathbf{Q}_{u}^{-1},
$$

where $\boldsymbol{\Lambda}_{f}$ is a diagonal matrix with the (unimodular) eigenvalues $\lambda_{n}$ of $\mathbf{T}_{u}$ on its main diagonal and where the columns of $\mathbf{Q}_{u}$ are actually the eigenvectors of $\mathbf{T}_{u}$. The subscript $u$ in connection to a matrix will throughout be used to denote that we are dealing with the unimodular case; the subscript $f$ will be used if the form of the matrix to which it is attached is in agreement with the more special case of a fractional Fourier transformer.

We first remark that if $\lambda$ is an eigenvalue of a real symplectic matrix $\mathbf{T}$, then $\lambda^{*}, 1 / \lambda$, and $1 / \lambda^{*}$ are eigenvalues, too; as usual, complex conjugation is denoted by the superscript ${ }^{*}$. Indeed, from the realness of $\mathbf{T}$, we conclude that the characteristic equation $\operatorname{det}(\mathbf{T}-\lambda \mathbf{I})=0$ has real coefficients and that the eigenvalues are thus real or come in complex conjugated pairs: If $\lambda$ is an eigenvalue, then $\lambda^{*}$ is an eigenvalue, too. Moreover, from the symplecticity condition of Eq. (2) we get

$$
\begin{aligned}
\operatorname{det}\left(\mathbf{T}^{-1}-\lambda \mathbf{I}\right) & =\operatorname{det}\left(\mathbf{J} \mathbf{T}^{t} \mathbf{J}-\lambda \mathbf{I}\right)=\operatorname{det}\left[\mathbf{J}\left(\mathbf{T}^{t}-\lambda \mathbf{I}\right) \mathbf{J}\right] \\
& =\operatorname{det}\left(\mathbf{T}^{t}-\lambda \mathbf{I}\right)=\operatorname{det}(\mathbf{T}-\lambda \mathbf{I}),
\end{aligned}
$$

and we conclude that if $\lambda$ is an eigenvalue, then $1 / \lambda$ is an eigenvalue, too. So for real symplectic matrices, the eigenvalues come in complex quartets (if they are not unimodular and not real), or in complex conjugated pairs (if they are unimodular, but not real), or in real pairs (in particular, they are double if equal to +1 or -1 ).

Let $\mathbf{q}_{n}$ be a (complex-valued) eigenvector of the symplectic matrix $\mathbf{T}$ with (complex-valued) eigenvalue $\lambda_{n}$ : $\mathbf{T} \mathbf{q}_{n}=\lambda_{n} \mathbf{q}_{n}$. Note that in the context of this paper, which deals with diagonalizable matrices, we may safely assume that the set of eigenvectors $\mathbf{q}_{n}$ has full rank. We now consider the inner product $\mathbf{q}_{n}^{t} \mathbf{J} \mathbf{q}_{m}=-\mathbf{q}_{m}^{t} \mathbf{J} \mathbf{q}_{n}$ and get

$$
\lambda_{n} \mathbf{q}_{n}^{t} \mathbf{J} \mathbf{q}_{m} \lambda_{m}=\left(\mathbf{T} \mathbf{q}_{n}\right)^{t} \mathbf{J}\left(\mathbf{T} \mathbf{q}_{m}\right)=\mathbf{q}_{n}^{t}\left(\mathbf{T}^{t} \mathbf{J} \mathbf{T}\right) \mathbf{q}_{m}=\mathbf{q}_{n}^{t} \mathbf{J} \mathbf{q}_{m},
$$

where we have used the symplecticity property $\mathbf{T}^{t} \mathbf{J T}=\mathbf{J}$ [see Eq. (2)]; hence,

$$
\left(\lambda_{n} \lambda_{m}-1\right) \mathbf{q}_{n}^{t} \mathbf{J} \mathbf{q}_{m}=0 .
$$

We conclude that the inner product $\mathbf{q}_{n}^{t} \mathbf{J} \mathbf{q}_{m}$ vanishes, except in the case of two partner eigenvectors $\mathbf{q}_{n}$ and $\mathbf{q}_{m}$ $=\widetilde{\mathbf{q}}_{n}$, i.e., the pair of eigenvectors $\mathbf{q}_{n}$ and $\widetilde{\mathbf{q}}_{n}$ with eigenvalues $\lambda_{n}$ and $\tilde{\lambda}_{n}$ that are each others' inverses: $\lambda_{n} \widetilde{\lambda}_{n}=1$. Again, in the context of this paper, we need not consider the particular case that both $\mathbf{q}_{n}^{t} \mathbf{J} \widetilde{\mathbf{q}}_{n}$ and $\lambda_{n} \widetilde{\lambda}_{n}-1$ vanish simultaneously: $\mathbf{q}_{n}^{t} \mathbf{J} \widetilde{\mathbf{q}}_{n}=0$ would imply that $\widetilde{\mathbf{q}}_{n}$ is a linear combination of all the other eigenvectors, in which case the set of eigenvectors would not have full rank, and the matrix $\mathbf{T}$ would not be diagonalizable.

Since we have freedom in choosing the lengths of the eigenvectors, we may choose them such that the inner product of each pair of partner eigenvectors becomes $-i: \mathbf{q}_{n}^{t} \mathbf{J} \widetilde{\mathbf{q}}_{n}=-i$. With a proper ordering of the eigenvalues into an eigenvalue matrix $\boldsymbol{\Lambda}$ as

$$
\Lambda=\left[\begin{array}{cc}
\Delta & \mathbf{0} \\
\mathbf{0} & \boldsymbol{\Delta}^{-1}
\end{array}\right]
$$

and with the corresponding ordering of the eigenvectors as column vectors in an eigenvector matrix $\mathbf{Q}$, both the eigenvalue matrix $\boldsymbol{\Lambda}$ and the eigenvector matrix $\mathbf{Q}$ will then be symplectic: $\boldsymbol{\Lambda}^{t} \mathbf{J} \Lambda=\mathbf{J}$ and $\mathbf{Q}^{t} \mathbf{J Q}=\mathbf{J}$. Note that symplecticity for complex symplectic matrices (like $\mathbf{Q}$ and $\Lambda$ ) is defined as usual by Eq. (2).

In the special case that the symplectic matrix $\mathbf{T}=\mathbf{T}_{u}$ is real and unimodular, the eigenvector $\mathbf{q}_{n}$ [with eigenvalue $\lambda_{n}=\exp \left(i \theta_{n}\right)$ and its partner $\widetilde{\mathbf{q}}_{n}$ [with eigenvalue $\tilde{\lambda}_{n}$ $\left.=1 / \lambda_{n}=\exp \left(-i \theta_{n}\right)=\lambda_{n}^{*}\right]$ are related to each other as $\tilde{\mathbf{q}}_{n}$ $\propto i \mathbf{q}_{n}^{*}$. This can directly be seen from the fact that (i) for a real symplectic matrix $\mathbf{T}$ we have the property that if $\mathbf{T} \mathbf{q}_{n}=\lambda_{n} \mathbf{q}_{n}$ then $\mathbf{T} \mathbf{q}_{n}^{*}=\lambda_{n}^{*} \mathbf{q}_{n}^{*}$, and (ii) for unimodularity we have the additional property that $\lambda_{n}^{*}=1 / \lambda_{n}=\tilde{\lambda}_{n}$. We assume again that the lengths of the eigenvectors have been chosen such that $\mathbf{q}_{n}^{t} \mathbf{J} \widetilde{\mathbf{q}}_{n}=-i$. The eigenvalue matrix $\boldsymbol{\Lambda}$ now takes the form

$$
\Lambda=\left[\begin{array}{cc}
\Delta & 0 \\
0 & \Delta^{-1}
\end{array}\right]=\left[\begin{array}{cc}
\Delta & 0 \\
0 & \Delta^{*}
\end{array}\right] \equiv \Lambda_{f},
$$

and the eigenvector matrix $\mathbf{Q}_{u}$ can be expressed as

$$
\mathbf{Q}_{u}=\frac{1}{\sqrt{2}}\left[\begin{array}{ll}
\mathbf{a}+i \mathbf{b} \mathbf{w}^{-2} & i\left(\mathbf{a}+i \mathbf{b} \mathbf{w}^{-2}\right)^{*} \mathbf{w}^{2} \\
\mathbf{c}+i \mathbf{d} \mathbf{w}^{-2} & i\left(\mathbf{c}+i \mathbf{d} \mathbf{w}^{-2}\right)^{*} \mathbf{w}^{2}
\end{array}\right]
$$

where $\mathbf{w}^{2}$ is an arbitrary diagonal matrix with positive real diagonal elements, which we will use for normalization purposes later.

\section{SIMILARITY TO A FRACTIONAL FOURIER TRANSFORMER}

The eigenvector matrix $\mathbf{Q}_{u}$ can be decomposed as

$$
\mathbf{Q}_{u}=\left[\begin{array}{ll}
\mathbf{a} & \mathbf{b} \\
\mathbf{c} & \mathbf{d}
\end{array}\right] \frac{1}{\sqrt{2}}\left[\begin{array}{cc}
\mathbf{I} & i \mathbf{w}^{2} \\
i \mathbf{w}^{-2} & \mathbf{I}
\end{array}\right] \equiv \mathbf{M F},
$$

with

$$
\begin{gathered}
\mathbf{M}=\left[\begin{array}{ll}
\mathbf{a} & \mathbf{b} \\
\mathbf{c} & \mathbf{d}
\end{array}\right], \\
\mathbf{F}=\frac{1}{\sqrt{2}}\left[\begin{array}{cc}
\mathbf{I} & i \mathbf{w}^{2} \\
i \mathbf{w}^{-2} & \mathbf{I}
\end{array}\right] .
\end{gathered}
$$

Symplecticity of the (real) matrix $\mathbf{M}$ follows directly from the symplecticity of the (complex) matrix $\mathbf{Q}_{u}$, using the symplecticity condition of Eq. (2). The matrix $\mathbf{F}$ contains as its columns the eigenvectors of the ray transformation matrix of a separable fractional Fourier transformer; 
moreover it is the (complex!) symplectic matrix that corresponds to the Bargmann-Segal transform, ${ }^{18,19}$ [see also Ref. 15, p. 282, Remark: Complex canonical transforms, Eq. (C.44)], which maps $\mathcal{L}^{2}$-space into Fock space and which bridges Fourier transformers and scale changers (Ref. 15, pp. 352-353, Remark: Fourier basis of aberrations of $F^{\alpha}$ transformers).

Using Eq. (16), the decomposition of Eq. (9) can be expressed in the form

$$
\mathbf{T}_{u}=\mathbf{M F} \Lambda_{f} \mathbf{F}^{-1} \mathbf{M}^{-1} \equiv \mathbf{M} \mathbf{T}_{f} \mathbf{M}^{-1},
$$

with

$$
\mathbf{T}_{f}=\mathbf{F} \Lambda_{f} \mathbf{F}^{-1}=\left[\begin{array}{cc}
\frac{\Delta+\boldsymbol{\Delta}^{*}}{2} & \frac{\boldsymbol{\Delta}-\boldsymbol{\Delta}^{*}}{2 i} \mathbf{w}^{2} \\
-\frac{\boldsymbol{\Delta}-\boldsymbol{\Delta}^{*}}{2 i} \mathbf{w}^{-2} & \frac{\boldsymbol{\Delta}+\boldsymbol{\Delta}^{*}}{2}
\end{array}\right]
$$

Since each diagonal element of the diagonal matrix $\Delta$ is unimodular, $\lambda_{n}=\exp \left(i \theta_{n}\right)=\cos \theta_{n}+i \sin \theta_{n}$, the submatrices $\left(\boldsymbol{\Delta}+\boldsymbol{\Delta}^{*}\right) / 2$ and $\left(\boldsymbol{\Delta}-\boldsymbol{\Delta}^{*}\right) / 2 i$ of $\mathbf{T}_{f}$ are diagonal matrices with elements $\cos \theta_{n}$ and $\sin \theta_{n}$, respectively. And when we combine the angles $\theta_{n}$ into a diagonal matrix $\boldsymbol{\Theta}$, we may express $\mathbf{T}_{f}$ as

$$
\mathbf{T}_{f}=\left[\begin{array}{cc}
\mathbf{w} & \mathbf{0} \\
\mathbf{0} & \mathbf{w}^{-1}
\end{array}\right]\left[\begin{array}{cc}
\cos \Theta & \sin \Theta \\
-\sin \Theta & \cos \Theta
\end{array}\right]\left[\begin{array}{cc}
\mathbf{w}^{-1} & \mathbf{0} \\
\mathbf{0} & \mathbf{w}
\end{array}\right] .
$$

From the latter expression we conclude that $\mathbf{T}_{f}$ $=\mathbf{T}_{f}\left(\theta_{1}, \theta_{2}, \ldots\right)$ is a separable fractional Fourier transformer (with scaling) with fractional angles $\theta_{1}, \theta_{2}, \ldots$, corresponding to rotations (with scaling) in phase space. We finally conclude that any lossless unimodular first-order optical system (with a diagonalizable ray transformation matrix $\mathbf{T}_{u}$ ) is similar (in the sense of matrix similarity: $\mathbf{T}_{u}=\mathbf{M} \mathbf{T}_{f} \mathbf{M}^{-1}$ ) to a separable fractional Fourier transformer (with scaling), where the fractional angles $\theta_{n}$ correspond to the phase angles of the unimodular eigenvalues: $\lambda_{n}=\exp \left(i \theta_{n}\right)$.

\section{SOME EXAMPLES}

Before we continue the development of our theory in Section 7, we first consider some examples to elucidate the concepts introduced so far.

\section{A. One-Dimensional Case}

In the one-dimensional case [with two unimodular eigenvalues $\exp ( \pm i \theta)$ and thus $|a+d| \leqslant 2$ ] we can formulate explicitly the expression

$$
\begin{aligned}
{\left[\begin{array}{ll}
a & b \\
c & d
\end{array}\right] } & =\left[\begin{array}{cc}
\cos \theta+g w^{2} \sin \theta & w^{2} \sin \theta \\
-\left(g^{2} w^{2}+w^{-2}\right) \sin \theta & \cos \theta-g w^{2} \sin \theta
\end{array}\right] \\
& =\left[\begin{array}{cr}
1 & 0 \\
-g & 1
\end{array}\right]\left[\begin{array}{cc}
\cos \theta & w^{2} \sin \theta \\
-w^{-2} \sin \theta & \cos \theta
\end{array}\right]\left[\begin{array}{ll}
1 & 0 \\
g & 1
\end{array}\right]
\end{aligned}
$$

for the decomposition that results after the similarity transformation described above [see Eq. (19)], where $\theta$ and $w^{2}$ follow from $a+d=2 \cos \theta$ and $b=w^{2} \sin \theta$, and $g$ fol- lows from $a-d=2 g b$. Note that this decomposition (with 3 degrees of freedom) corresponds to a scaled fractional Fourier transformer [see Eq. (5)] embedded in between two lenses with opposite focal lengths [see the central matrix in expression (4)].

Although a bit beyond the scope of this paper, we remark that in the nonunimodular case [with eigenvalues $+\exp ( \pm \theta)$ or $-\exp ( \pm \theta)$, and thus $|a+d| \geqslant 2]$, the ray transformation matrix can be decomposed in a more or less similar form as

$$
\begin{aligned}
{\left[\begin{array}{ll}
a & b \\
c & d
\end{array}\right]=} & {\left[\begin{array}{cc}
1 & 0 \\
-g & 1
\end{array}\right]\left[\begin{array}{cc} 
\pm \cosh \theta & \pm w^{2} \sinh \theta \\
\pm w^{-2} \sinh \theta & \pm \cosh \theta
\end{array}\right] } \\
& \times\left[\begin{array}{ll}
1 & 0 \\
g & 1
\end{array}\right],
\end{aligned}
$$

where $\theta$ and $w^{2}$ (together with the proper choice of the sign) follow now from $a+d= \pm 2 \cosh \theta$ and $b= \pm w^{2} \sinh \theta$. The decomposition is now built around a hyperbolic expander, whose optical realization is similar to that of the fractional Fourier transformer setup (a) mentioned in Section 2, but with a concave lens instead of a convex one (see also Ref. 15, p. 183, Example: Hyperbolic expanders).

\section{B. Orthosymplectic Class}

If the ray transformation matrix is not only real symplectic but also orthogonal, we call the system orthosymplectic. ${ }^{15}$ The ray transformation matrix $\mathbf{T}$ 。 such an orthosymplectic system takes the general form

$$
\mathbf{T}_{\circ}=\left[\begin{array}{cc}
\mathbf{w} & \mathbf{0} \\
\mathbf{0} & \mathbf{w}^{-1}
\end{array}\right]\left[\begin{array}{cc}
\mathbf{X} & \mathbf{Y} \\
-\mathbf{Y} & \mathbf{X}
\end{array}\right]\left[\begin{array}{cc}
\mathbf{w}^{-1} & 0 \\
\mathbf{0} & \mathbf{w}
\end{array}\right],
$$

where the two matrices $\mathbf{X}$ and $\mathbf{Y}$ can be combined into one complex matrix $\mathbf{U}=\mathbf{X}+i \mathbf{Y}$ that is unitary: $\mathbf{U}^{\dagger}=\mathbf{U}^{-1}$. We remark that the separable fractional Fourier transformer [see Eq. (21)] is clearly orthosymplectic, with $\mathbf{X}_{f}=\cos \Theta$ and $\mathbf{Y}_{f}=\sin \boldsymbol{\Theta}$, and $\mathbf{U}_{f}=\exp (i \boldsymbol{\Theta})$ [see also Ref. 15, Section 10.3.2, Eq. (10.31)]. For the sake of convenience, we will choose $\mathbf{w}=\mathbf{I}$ in the remainder of this section.

It is well known that a unitary matrix has unimodular eigenvalues and that it can be diagonalized (Ref. 14, Chap. 13). Moreover, it is not difficult to show that when the unitary matrix $\mathbf{U}$ is diagonalized with a matrix $\mathbf{P}$ to $\mathbf{U}=\mathbf{P} \Delta \mathbf{P}^{-1}$, the corresponding orthosymplectic matrix can be diagonalized as

$$
\mathbf{T}_{\circ}=\left[\begin{array}{cc}
\mathbf{P} & i \mathbf{P}^{*} \\
i \mathbf{P} & \mathbf{P}^{*}
\end{array}\right]\left[\begin{array}{cc}
\boldsymbol{\Delta} & \mathbf{0} \\
\mathbf{0} & \boldsymbol{\Delta}^{*}
\end{array}\right]\left[\begin{array}{cc}
\mathbf{P} & i \mathbf{P}^{*} \\
i \mathbf{P} & \mathbf{P}^{*}
\end{array}\right]^{-1} \equiv \mathbf{Q}_{\circ} \boldsymbol{\Lambda}_{f} \mathbf{Q}_{\circ}^{-1} .
$$

We remark that the class of orthosymplectic systems is an important subclass of unimodular systems. Moreover, it will be clear that in the case of orthosymplectic systems, it is much easier to work with the unitary $D \times D$ matrix $\mathbf{U}$ than to work with the orthosymplectic $2 D \times 2 D$ matrix $\mathbf{T}_{\text {。. }}$

The matrix $\mathbf{P}$ that diagonalizes the unitary matrix $\mathbf{U}, \mathbf{U}=\mathbf{P} \Delta \mathbf{P}^{-1}$, can itself be made unitary, and we conclude that all three matrices in the cascade $\mathbf{P} \Delta \mathbf{P}^{-1}$ are unitary. While the unimodular matrix $\Delta$ is the unitary representation of a separable fractional Fourier transformer, the matrix $\mathbf{P}$ can also be considered as the unitary represen- 
tation of an orthosymplectic system that is optically realizable, i.e., a system whose symplectic ray transformation matrix is real. We thus conclude that for orthosymplectic systems there is a direct way from the decomposition $\mathbf{U}$ $=\mathbf{P} \Delta \mathbf{P}^{-1}$ to $\mathbf{T}_{\circ}=\mathbf{M}_{\circ} \mathbf{T}_{f} \mathbf{M}_{\circ}^{-1}$, with $\mathbf{M}_{\circ}$ the orthosymplectic system whose unitary representation reads $\mathbf{P}$, and that we do not need the intermediate decomposition [Eq. (25)].

\section{Some Two-Dimensional Examples from the Orthosymplectic Class}

In the two-dimensional case, with $\mathbf{r}=(x, y)^{t}$ and $\mathbf{p}$ $=\left(p_{x}, p_{y}\right)^{t}$, basic members of the orthosymplectic class are, apart from the two-dimensional separable fractional Fourier transformer with ray transformation matrix $\mathbf{T}_{f}\left(\theta_{x}, \theta_{y}\right)$ and with the unitary representation

$$
\mathbf{U}_{f}\left(\theta_{x}, \theta_{y}\right)=\left[\begin{array}{cc}
\exp \left(i \theta_{x}\right) & 0 \\
0 & \exp \left(i \theta_{y}\right)
\end{array}\right]
$$

the rotator (also called image gyrator ${ }^{20}$ ) and the gyrator (also called cross gyrator ${ }^{20}$ ).

These two systems can be described by the ray transformation matrices

$$
\begin{aligned}
\mathbf{T}_{r}(\theta) & =\left[\begin{array}{cccc}
\cos \theta & \sin \theta & 0 & 0 \\
-\sin \theta & \cos \theta & 0 & 0 \\
0 & 0 & \cos \theta & \sin \theta \\
0 & 0 & -\sin \theta & \cos \theta
\end{array}\right], \\
\mathbf{T}_{g}(\theta) & =\left[\begin{array}{cccc}
\cos \theta & 0 & 0 & \sin \theta \\
0 & \cos \theta & \sin \theta & 0 \\
0 & -\sin \theta & \cos \theta & 0 \\
-\sin \theta & 0 & 0 & \cos \theta
\end{array}\right]
\end{aligned}
$$

[see also Ref. 20, Eqs. (49) and (58)], as well as by the unitary matrices

$$
\begin{aligned}
& \mathbf{U}_{r}(\theta)=\left[\begin{array}{cc}
\cos \theta & \sin \theta \\
-\sin \theta & \cos \theta
\end{array}\right], \\
& \mathbf{U}_{g}(\theta)=\left[\begin{array}{cc}
\cos \theta & i \sin \theta \\
i \sin \theta & \cos \theta
\end{array}\right],
\end{aligned}
$$

respectively. We remark that a rotator with rotation angle $\theta$ produces a rotation through the angle $\theta$, both for the spatial variables $(x, y)$ and the spatial-frequency variables $\left(p_{x}, p_{y}\right)$, while a gyrator produces a rotation for the mixedvariables combinations $\left(x, p_{y}\right)$ and $\left(y, p_{x}\right)$.

When we bring the unitary matrices $\mathbf{U}_{r}(\theta)$ and $\mathbf{U}_{g}(\theta)$ in diagonal form by means of the unitary matrices $\mathbf{P}_{r}$ and $\mathbf{P}_{g}, \mathbf{U}_{r}(\theta)=\mathbf{P}_{r} \Delta_{r} \mathbf{P}_{r}^{-1}$ and $\mathbf{U}_{g}(\theta)=\mathbf{P}_{g} \Delta_{g} \mathbf{P}_{g}^{-1}$, we get

$$
\begin{aligned}
\mathbf{P}_{r} & =\frac{1}{\sqrt{2}}\left[\begin{array}{ll}
1 & i \\
i & 1
\end{array}\right]\left[\begin{array}{cc}
\exp (i \alpha) & 0 \\
0 & \exp (i \beta)
\end{array}\right] \\
& =\mathbf{U}_{g}(\pi / 4) \mathbf{U}_{f}(\alpha, \beta)=\mathbf{P}_{r}(\alpha, \beta),
\end{aligned}
$$

$$
\begin{aligned}
\mathbf{P}_{g} & =\frac{1}{\sqrt{2}}\left[\begin{array}{cc}
1 & -1 \\
1 & 1
\end{array}\right]\left[\begin{array}{cc}
\exp (i \alpha) & 0 \\
0 & \exp (i \beta)
\end{array}\right] \\
& =\mathbf{U}_{r}(-\pi / 4) \mathbf{U}_{f}(\alpha, \beta)=\mathbf{P}_{g}(\alpha, \beta),
\end{aligned}
$$

with arbitrary values of $\alpha$ and $\beta$, and

$$
\boldsymbol{\Delta}_{r}=\boldsymbol{\Delta}_{g}=\left[\begin{array}{cc}
\exp (i \theta) & 0 \\
0 & \exp (-i \theta)
\end{array}\right]=\mathbf{U}_{f}(\theta,-\theta) \text {. }
$$

With $\mathbf{P}_{r}(\alpha, \beta), \mathbf{P}_{g}(\alpha, \beta)$, and $\mathbf{U}_{f}(\theta,-\theta)$ the unitary representations of the orthosymplectic systems $\mathbf{M}_{r}, \mathbf{M}_{g}$, and $\mathbf{T}_{f}$, respectively, we get

$$
\begin{aligned}
\mathbf{M}_{r} & =\mathbf{M}_{r}(\alpha, \beta) \\
& =\frac{1}{\sqrt{2}}\left[\begin{array}{cccc}
\cos \alpha & -\sin \beta & \sin \alpha & \cos \beta \\
-\sin \alpha & \cos \beta & \cos \alpha & \sin \beta \\
-\sin \alpha & -\cos \beta & \cos \alpha & -\sin \beta \\
-\cos \alpha & -\sin \beta & -\sin \alpha & \cos \beta
\end{array}\right], \\
\mathbf{M}_{g} & =\mathbf{M}_{g}(\alpha, \beta) \\
& =\frac{1}{\sqrt{2}}\left[\begin{array}{cccc}
\cos \alpha & -\cos \beta & \sin \alpha & -\sin \beta \\
\cos \alpha & \cos \beta & \sin \alpha & \sin \beta \\
-\sin \alpha & \sin \beta & \cos \alpha & -\cos \beta \\
-\sin \alpha & -\sin \beta & \cos \alpha & \cos \beta
\end{array}\right]
\end{aligned}
$$

[see also Ref. 20, Eq. (63)],

$$
\mathbf{T}_{f}=\mathbf{T}_{f}(\theta,-\theta)=\left[\begin{array}{cccc}
\cos \theta & 0 & \sin \theta & 0 \\
0 & \cos \theta & 0 & -\sin \theta \\
-\sin \theta & 0 & \cos \theta & 0 \\
0 & \sin \theta & 0 & \cos \theta
\end{array}\right]
$$

We remark that $\mathbf{T}_{f}(\theta,-\theta)$ corresponds to a separable fractional Fourier transformer with fractional angles $\theta$ and $-\theta$ in the $x$ and $y$ directions, respectively. Moreover, $\mathbf{M}_{r}(\alpha, \beta)$ corresponds to a cascade of a $(\pi / 4)$ gyrator $\mathbf{T}_{g}(\pi / 4)$ and a separable fractional Fourier transformer $\mathbf{T}_{f}(\alpha, \beta)$ [see Eq. (31)], while $\mathbf{M}_{g}(\alpha, \beta)$ corresponds to a cascade of a $(-\pi / 4)$ rotator $\mathbf{T}_{r}(-\pi / 4)$ and such a separable fractional Fourier transformer [see Eq. (32)]. We thus have the relations

$$
\begin{aligned}
& \mathbf{T}_{r}(\theta)=\mathbf{T}_{g}(\pi / 4) \mathbf{T}_{f}(\theta,-\theta) \mathbf{T}_{g}(-\pi / 4), \\
& \mathbf{T}_{g}(\theta)=\mathbf{T}_{r}(-\pi / 4) \mathbf{T}_{f}(\theta,-\theta) \mathbf{T}_{r}(\pi / 4),
\end{aligned}
$$

where we have used the fact that the two separable fractional Fourier transformers $\mathbf{T}_{f}(\alpha, \beta)$ and $\mathbf{T}_{f}^{-1}(\alpha, \beta)$ that embed $\mathbf{T}_{f}(\theta,-\theta)$ cancel each others' operations and might as well be omitted. Note the correspondence between Eq. (38) and Eq. (62) of Ref. 20, with $\mathbf{T}_{r}(\theta) \sim \mathcal{G}(\theta), \mathbf{T}_{g}(\theta)$ $\sim \mathcal{X}(\theta)$, and $\mathbf{T}_{f}(\theta,-\theta) \sim \mathcal{Y}(\theta)$.

For the separable fractional Fourier transformer $\mathbf{T}_{f}\left(\theta_{x}, \theta_{y}\right)$ itself, with the unitary representation $\mathbf{U}_{f}\left(\theta_{x}, \theta_{y}\right)$ 
that has already a diagonal form [see Eq. (26)], we proceed analogously. From the initial decomposition

$$
\mathbf{U}_{f}\left(\theta_{x}, \theta_{y}\right)=\mathbf{P}_{f} \boldsymbol{\Delta}_{f} \mathbf{P}_{f}^{-1} \equiv \mathbf{U}_{f}(\alpha, \beta) \mathbf{U}_{f}\left(\theta_{x}, \theta_{y}\right) \mathbf{U}_{f}^{-1}(\alpha, \beta),
$$

we are immediately led to the final decomposition

$$
\mathbf{T}_{f}\left(\theta_{x}, \theta_{y}\right)=\mathbf{M}_{f} \mathbf{T}_{f} \mathbf{M}_{f}^{-1} \equiv \mathbf{T}_{f}(\alpha, \beta) \mathbf{T}_{f}\left(\theta_{x}, \theta_{y}\right) \mathbf{T}_{f}^{-1}(\alpha, \beta) .
$$

We remark that $\mathbf{M}_{f}=\mathbf{T}_{f}(\alpha, \beta)$ is itself a separable fractional Fourier transformer again, with arbitrary angles $\alpha$ and $\beta$, and that the final cascade of Eq. (40) consists of three commuting separable fractional Fourier transformers; moreover, $\mathbf{T}_{f}(\alpha, \beta)$ and $\mathbf{T}_{f}^{-1}(\alpha, \beta)$ obviously cancel each others' operations.

\section{EIGENFUNCTIONS OF UNIMODULAR SYSTEMS}

In this section we use the decomposition of Eq. (19) developed in Section 5, $\mathbf{T}_{u}=\mathbf{M} \mathbf{T}_{f} \mathbf{M}^{-1}$ to find explicit expressions for the eigenfunctions that belong to the unimodular system described by the ray transformation matrix $\mathbf{T}_{u}$. For convenience, we restrict ourselves to the two-dimensional case and write $\mathbf{r}=(x, y)^{t}$; the ray transformation matrices are now $4 \times 4$ matrices. The extension to more dimensions, if necessary, is straightforward. Moreover, we will use normalized variables $\mathbf{r}=: \mathbf{w}^{-1} \mathbf{r}$ and $\mathbf{p}=\mathbf{w} \mathbf{p}$, and, consequently, normalized versions of the ray transformation matrices $\mathbf{T}, \mathbf{T}_{u}, \mathbf{T}_{f}$, and $\mathbf{M}$ :

$$
\mathbf{T}=:\left[\begin{array}{cc}
\mathbf{w}^{-1} & \mathbf{0} \\
\mathbf{0} & \mathbf{w}
\end{array}\right] \mathbf{T}\left[\begin{array}{cc}
\mathbf{w} & \mathbf{0} \\
\mathbf{0} & \mathbf{w}^{-1}
\end{array}\right] .
$$

Note that $\mathbf{T}_{f}$ then takes the form

$$
\mathbf{T}_{f}=:\left[\begin{array}{cc}
\cos \Theta & \sin \Theta \\
-\sin \Theta & \cos \Theta
\end{array}\right]
$$

[see Eq. (21)], which is the usual (normalized) representation of a separable fractional Fourier transformer, and that $\mathbf{M}$ takes the form

$$
\left[\begin{array}{ll}
\mathbf{a} & \mathbf{b} \\
\mathbf{c} & \mathbf{d}
\end{array}\right]=:\left[\begin{array}{cc}
\mathbf{w}^{-1} & \mathbf{0} \\
\mathbf{0} & \mathbf{w}
\end{array}\right]\left[\begin{array}{ll}
\mathbf{a} & \mathbf{b} \\
\mathbf{c} & \mathbf{d}
\end{array}\right]\left[\begin{array}{cc}
\mathbf{w} & \mathbf{0} \\
\mathbf{0} & \mathbf{w}^{-1}
\end{array}\right] .
$$

We now determine the eigenfunctions $\Phi_{n}(\mathbf{r})$ of the linear canonical integral transformation $f_{o}\left(\mathbf{r}_{o}\right)=\mathcal{R}_{u}\left[f_{i}\left(\mathbf{r}_{i}\right)\right]\left(\mathbf{r}_{o}\right)$ that corresponds to a unimodular system. Eigenfunctions are defined in the usual way,

$$
\Phi_{n}\left(\mathbf{r}_{o}\right)=\mu_{n} \mathcal{R}_{u}\left[\Phi_{n}\left(\mathbf{r}_{i}\right)\right]\left(\mathbf{r}_{o}\right),
$$

where $\mu_{n}$ is the corresponding (unimodular) eigenvalue. We recall ${ }^{11-13}$ that for the one-dimensional fractional Fourier transformer with fractional angle $\theta$, the eigenfunctions are the Hermite-Gauss functions

$$
\mathcal{H}_{n}(x)=2^{1 / 4}\left(2^{n} n !\right)^{-1 / 2} H_{n}(x \sqrt{2 \pi}) \exp \left(-\pi x^{2}\right),
$$

with $H_{n}(\cdot)$ the Hermite polynomials (Ref. 14, Section 21); the corresponding eigenvalues read $\mu_{n}=\exp (-i n \theta)$.

Since $\mathbf{M}$ is a real symplectic ray transformation matrix, an orthonormal set of eigenfunctions of the system $\mathbf{T}_{u}$ $=\mathbf{M} \mathbf{T}_{f} \mathbf{M}^{-1}$ can be given explicitly, based on the fact that the separable Hermite-Gauss functions $\mathcal{H}_{n, m}(\mathbf{r})$ $=\mathcal{H}_{n}(x) \mathcal{H}_{m}(y)$ are eigenfunctions of the separable fractional Fourier transformer $\mathbf{T}_{f}\left(\theta_{x}, \theta_{y}\right)$ with fractional angles $\theta_{x}$ and $\theta_{y}$ in the $x$ and $y$ directions, respectively. These eigenfunctions of the system $\mathbf{T}_{u}$, which we denote by $\Phi_{n}(\mathbf{r})=\mathcal{H}_{n, m}^{\mathbf{M}}(\mathbf{r})$, follow from studying the propagation of the separable Hermite-Gauss functions $\mathcal{H}_{n, m}(\mathbf{r})$ $=\mathcal{H}_{n}(x) \mathcal{H}_{m}(y)$ through the first-order optical subsystem with ray transformation matrix $\mathbf{M}$, and are most easily determined by their generating function ${ }^{21,22}$

$$
\begin{aligned}
& \frac{2^{1 / 2}}{\sqrt{\operatorname{det}(\mathbf{a}+i \mathbf{b})}} \exp \left[-\mathbf{s}^{t}(\mathbf{a}+i \mathbf{b})^{-1}(\mathbf{a}-i \mathbf{b}) \mathbf{s}\right. \\
& \left.\quad+2 \mathbf{s}^{t}(\mathbf{a}+i \mathbf{b})^{-1} \mathbf{r} \sqrt{2 \pi}-\pi \mathbf{r}^{t}(\mathbf{d}-i \mathbf{c})(\mathbf{a}+i \mathbf{b})^{-1} \mathbf{r}\right] \\
& =\sum_{n=0}^{\infty} \sum_{m=0}^{\infty} \mathcal{H}_{n, m}^{\mathbf{M}}(\mathbf{r})\left(\frac{2^{n+m}}{n ! m !}\right)^{1 / 2} s_{x}^{n} s_{y}^{m},
\end{aligned}
$$

with $\mathbf{s}=\left(s_{x}, s_{y}\right)^{t}$, where $\mathbf{a}, \mathbf{b}, \mathbf{c}$, and $\mathbf{d}$ are the submatrices that constitute the subsystem's ray transformation matrix M. An explicit form for these eigenfunctions has also been derived ${ }^{22}$ and reads

$$
\begin{aligned}
\mathcal{H}_{n, m}^{\mathbf{M}}(\mathbf{r})= & \frac{2^{1 / 2}}{2^{n+m} \sqrt{\pi^{n+m} n ! m !} \sqrt{\operatorname{det}(\mathbf{a}+i \mathbf{b})}} \\
& \times \mathcal{P}_{x}^{n} \mathcal{P}_{y}^{m} \exp \left[-\pi \mathbf{r}^{t}(\mathbf{d}-i \mathbf{c})(\mathbf{a}+i \mathbf{b})^{-1} \mathbf{r}\right],
\end{aligned}
$$

with the operators $\mathcal{P}_{x}$ and $\mathcal{P}_{y}$ defined as

$$
\left[\begin{array}{l}
\mathcal{P}_{x} \\
\mathcal{P}_{y}
\end{array}\right]=2 \pi \mathbf{V}\left[\begin{array}{l}
x \\
y
\end{array}\right]-\mathbf{Z}\left[\begin{array}{l}
\partial / \partial x \\
\partial / \partial y
\end{array}\right],
$$

where the matrices $\mathbf{V}$ and $\mathbf{Z}$ are related to $\mathbf{M}$ via

$$
\begin{gathered}
\mathbf{V}=(\mathbf{a}-i \mathbf{b})^{t}\left[(\mathbf{d}-i \mathbf{c})(\mathbf{a}+i \mathbf{b})^{-1}\right]^{*} \\
\mathbf{Z}=(\mathbf{a}-i \mathbf{b})^{t}
\end{gathered}
$$

We remark that the operators $\mathcal{P}_{x}$ and $\mathcal{P}_{y}$ commute since $\mathbf{Z} \mathbf{V}^{t}=\mathbf{V Z} \mathbf{Z}^{t}$, which is a direct consequence of the symplecticity of $\mathbf{M}$.

That $\mathcal{H}_{n, m}^{\mathbf{M}}(\mathbf{r})$ are eigenfunctions of the first-order optical system described by $\mathbf{T}_{u}=\mathbf{M} \mathbf{T}_{f} \mathbf{M}^{-1}$ can easily be seen as follows. ${ }^{21}$ If we apply the functions $\mathcal{H}_{n, m}^{\mathbf{M}}(\mathbf{r})$ to the cascade $\mathbf{M} \mathbf{T}_{f} \mathbf{M}^{-1}$, the subsystem $\mathbf{M}^{-1}$ will transform them into Hermite-Gauss functions $\mathcal{H}_{n, m}(\mathbf{r})$, which then pass the separable fractional Fourier transformer $\mathbf{T}_{f}$ only to be multiplied by a phase factor. The subsystem $\mathbf{M}$ finally transforms the Hermite-Gauss functions back into $\mathcal{H}_{n, m}^{\mathbf{M}}(\mathbf{r})$. Note that the separable Hermite-Gauss functions $\mathcal{H}_{n, m}(\mathbf{r})=\mathcal{H}_{n}(x) \mathcal{H}_{m}(y)$ themselves arise for $\mathbf{M}=\mathbf{I}$.

In the special case that $\mathbf{M}$ corresponds to an orthosymplectic system ${ }^{15}$ with unitary representation $\mathbf{U}=\mathbf{a}+i \mathbf{b}$ $=\mathbf{d}-i \mathbf{c}$, the exponent in the generating function of Eq. (46) reduces to $\exp \left[-\left(\mathbf{U}^{*} \mathbf{s}\right)^{t}\left(\mathbf{U}^{*} \mathbf{s}\right)+2\left(\mathbf{U}^{*} \mathbf{s}\right)^{t} \mathbf{r} \sqrt{2 \pi}-\pi \mathbf{r}^{t} \mathbf{r}\right]$, from which we conclude that an orthosymplectic system does not change the Gaussian dependence on $\mathbf{r}$ : $\exp \left(-\pi \mathbf{r}^{t} \mathbf{r}\right)$. This is also clear from the explicit form of Eq. (47), which now reads 


$$
\mathcal{H}_{n, m}^{\mathbf{M}}(\mathbf{r})=\frac{2^{1 / 2} \mathcal{P}_{x}^{n} \mathcal{P}_{y}^{m} \exp \left(-\pi \mathbf{r}^{t} \mathbf{r}\right)}{2^{n+m} \sqrt{\pi^{n+m} n ! m !} \sqrt{\operatorname{det} \mathbf{U}}}
$$

and where the unitary matrix $\mathbf{U}$ and the matrices $\mathbf{V}$ and $\mathbf{Z}$ that determine the operators $\mathcal{P}_{x}$ and $\mathcal{P}_{y}$ are related to each other by the relationship $\mathbf{V}=\mathbf{Z}=(\mathbf{a}-i \mathbf{b})^{t}=\mathbf{U}^{\dagger}=\mathbf{U}^{-1}$ [see Eqs. (49)].

\section{ALTERNATIVE DECOMPOSITION}

An alternative decomposition of $\mathbf{T}_{u}=\mathbf{M} \mathbf{T}_{f} \mathbf{M}^{-1}$ can be obtained when we represent the (normalized) symplectic matrix $\mathbf{M}$ by means of its Iwasawa decomposition. ${ }^{23}$ In particular we have (Ref. 15, Sections 9.5 and 10.2)

$$
\mathbf{M}=\left[\begin{array}{ll}
\mathbf{a} & \mathbf{b} \\
\mathbf{c} & \mathbf{d}
\end{array}\right]=\left[\begin{array}{cc}
\mathbf{I} & \mathbf{0} \\
-\mathbf{G} & \mathbf{I}
\end{array}\right]\left[\begin{array}{cc}
\mathbf{S} & \mathbf{0} \\
\mathbf{0} & \mathbf{S}^{-1}
\end{array}\right]\left[\begin{array}{cc}
\mathbf{X} & \mathbf{Y} \\
-\mathbf{Y} & \mathbf{X}
\end{array}\right]
$$

where the first matrix represents a lens described by the symmetric matrix

$$
\mathbf{G}=-\left(\mathbf{c a}^{t}+\mathbf{d} \mathbf{b}^{t}\right)\left(\mathbf{a} \mathbf{a}^{t}+\mathbf{b b}^{t}\right)^{-1}
$$

the second matrix represents a magnifier described by the positive-definite symmetric matrix

$$
\mathbf{S}=\left(\mathbf{a a}^{t}+\mathbf{b b}^{t}\right)^{1 / 2},
$$

and the third matrix represents an orthosymplectic system described by the unitary matrix

$$
\mathbf{U}=\mathbf{X}+i \mathbf{Y}=\left(\mathbf{a a}^{t}+\mathbf{b b}^{t}\right)^{-1 / 2}(\mathbf{a}+i \mathbf{b}) .
$$

Substitution of the Iwasawa decomposition [Eq. (51)] into the expression $\mathbf{T}_{u}=\mathbf{M} \mathbf{T}_{f} \mathbf{M}^{-1}$ leads to

$$
\begin{aligned}
\mathbf{T}_{u}= & {\left[\begin{array}{cc}
\mathbf{I} & \mathbf{0} \\
-\mathbf{G} & \mathbf{I}
\end{array}\right]\left[\begin{array}{cc}
\mathbf{S} & \mathbf{0} \\
\mathbf{0} & \mathbf{S}^{-1}
\end{array}\right] } \\
& \times\left[\begin{array}{cc}
\mathbf{X} & \mathbf{Y} \\
-\mathbf{Y} & \mathbf{X}
\end{array}\right]\left[\begin{array}{cc}
\cos \boldsymbol{\Theta} & \sin \boldsymbol{\Theta} \\
-\sin \boldsymbol{\Theta} & \cos \boldsymbol{\Theta}
\end{array}\right]\left[\begin{array}{cc}
\mathbf{X}^{t} & -\mathbf{Y}^{t} \\
\mathbf{Y}^{t} & \mathbf{X}^{t}
\end{array}\right] \\
& \times\left[\begin{array}{cc}
\mathbf{S}^{-1} & \mathbf{0} \\
\mathbf{0} & \mathbf{S}
\end{array}\right]\left[\begin{array}{cc}
\mathbf{I} & \mathbf{0} \\
\mathbf{G} & \mathbf{I}
\end{array}\right] .
\end{aligned}
$$

The product of the three matrices in the middle of this expression,

$$
\left[\begin{array}{cc}
\mathbf{X} & \mathbf{Y} \\
-\mathbf{Y} & \mathbf{X}
\end{array}\right]\left[\begin{array}{cc}
\cos \boldsymbol{\Theta} & \sin \Theta \\
-\sin \Theta & \cos \Theta
\end{array}\right]\left[\begin{array}{cc}
\mathbf{X}^{t} & -\mathbf{Y}^{t} \\
\mathbf{Y}^{t} & \mathbf{X}^{t}
\end{array}\right]
$$

is actually a product of three orthosymplectic matrices. When we represent these orthosymplectic matrices by their unitary representations $\mathbf{U}=\mathbf{X}+i \mathbf{Y}, \exp (i \Theta)=\cos \Theta$ $+i \sin \Theta$, and $\mathbf{U}^{-1}=\mathbf{X}^{t}-i \mathbf{Y}^{t}$, respectively, we get the matrix product $\mathbf{U}_{\circ}=\mathbf{U} \exp (i \Theta) \mathbf{U}^{-1}$, which expresses the diagonalization of the unitary matrix $\mathbf{U}_{\circ}$. Since the matrix $\mathbf{U}_{\circ}=\mathbf{X}$ $+i \mathbf{Y}$ 。 can be considered again as the unitary representation of an orthosymplectic matrix, we are thus led to the alternative decomposition

$$
\begin{aligned}
\mathbf{T}_{u}= & {\left[\begin{array}{cc}
\mathbf{I} & \mathbf{0} \\
-\mathbf{G} & \mathbf{I}
\end{array}\right]\left[\begin{array}{cc}
\mathbf{S} & \mathbf{0} \\
\mathbf{0} & \mathbf{S}^{-1}
\end{array}\right]\left[\begin{array}{cc}
\mathbf{X}_{\circ} & \mathbf{Y}_{\circ} \\
-\mathbf{Y}_{\circ} & \mathbf{X}_{\circ}
\end{array}\right]\left[\begin{array}{cc}
\mathbf{S}^{-1} & \mathbf{0} \\
\mathbf{0} & \mathbf{S}
\end{array}\right] } \\
& \times\left[\begin{array}{cc}
\mathbf{I} & \mathbf{0} \\
\mathbf{G} & \mathbf{I}
\end{array}\right]
\end{aligned}
$$

The decomposition of Eq. (57) is different from the decomposition $\mathbf{T}_{u}=\mathbf{M} \mathbf{T}_{f} \mathbf{M}^{-1}$ that we had before and that in detail reads

$$
\mathbf{T}_{u}=\left[\begin{array}{ll}
\mathbf{a} & \mathbf{b} \\
\mathbf{c} & \mathbf{d}
\end{array}\right]\left[\begin{array}{cc}
\cos \Theta & \sin \Theta \\
-\sin \Theta & \cos \Theta
\end{array}\right]\left[\begin{array}{cc}
\mathbf{d}^{t} & -\mathbf{b}^{t} \\
-\mathbf{c}^{t} & \mathbf{a}^{t}
\end{array}\right] .
$$

In particular we note that the decomposition of Eq. (57) has less degrees of freedom than the decomposition of Eq. (58), but still enough to reach all possible real symplectic matrices with unimodular eigenvalues. For example, if we are dealing with $D$-dimensional position and direction vectors $\mathbf{r}$ and $\mathbf{p}$, the $2 D \times 2 D$-dimensional symplectic matrix $\mathbf{T}_{u}$ has $2 D^{2}+D$ degrees of freedom. This is the same number as we have in the decomposition of Eq. (57): $\left(D^{2}\right.$ $+D) / 2$ for the symmetric matrix $\mathbf{G}$ that describes the lens, $\left(D^{2}+D\right) / 2$ for the symmetric matrix $\mathbf{S}$ that describes the magnifier, and $D^{2}$ for the unitary matrix $\mathbf{X}_{\circ}+i \mathbf{Y}_{\circ}$ that describes the orthosymplectic system. The decomposition of Eq. (58) has $D$ more degrees of freedom, i.e., in addition to the $2 D^{2}+D$ degrees of freedom of the symplectic matrix $\mathbf{M}$, the $D$ phase angles $\theta_{n}$ of the unimodular eigenvalues $\lambda_{n}(n=1,2, \ldots, D)$. We thus conclude that the decomposition of Eq. (57) is better suited for the construction of unimodular first-order optical systems.

Note that in the one-dimensional case, the orthosymplectic matrix that appears in the Iwasawa decomposition of Eq. (51) is described by the scalar $u=(a+i b)\left(a^{2}\right.$ $\left.+b^{2}\right)^{-1 / 2}=\exp (i \theta)$, corresponding to a one-dimensional fractional Fourier transformer [see also Ref. 13, Sec. 9.7, in particular Eqs. (9.124)-(9.128)]. The decomposition of Eq. (57) then reduces to what has already been mentioned in Subsection 6.A.

\section{OPTICAL REALIZATION FOR THE CASE $D=2$}

Although the description of an optical realization is somewhat beyond the scope of this paper, we will present such a realization for the sake of completeness. We remark, however, that optical realizations of the subsystems that we will encounter can be found elsewhere as well. Note that in the case of $D=2$, we have $\mathbf{r}=(x, y)^{t}$, and the matrices $\mathbf{G}, \mathbf{S}, \mathbf{X}_{\circ}, \mathbf{Y}_{\circ}$, and $\mathbf{U}_{\circ}=\mathbf{X}_{\circ}+i \mathbf{Y}_{\circ}$ are $2 \times 2$ matrices.

It can be shown ${ }^{24}$ that in the special case of $D=2$, the orthosymplectic subsystem in the decomposition of Eq. (57), described by the unitary matrix $\mathbf{U}_{\circ}=\mathbf{X}_{\circ}+i \mathbf{Y}_{\circ}$, can be realized as a separable fractional Fourier transformer $\mathbf{U}_{f}\left(\gamma_{x}, \gamma_{y}\right)$

$$
\mathbf{U}_{f}\left(\gamma_{x}, \gamma_{y}\right)=\left[\begin{array}{cc}
\exp \left(i \gamma_{x}\right) & 0 \\
0 & \exp \left(i \gamma_{y}\right)
\end{array}\right],
$$

embedded in between two rotators $\mathbf{U}_{r}(\alpha)$ and $\mathbf{U}_{r}(\beta)$ [see also Ref. 15, Eq. (10.32)]. We thus have 
$\mathbf{U}_{\circ}=\mathbf{U}_{r}(\beta) \mathbf{U}_{f}\left(\gamma_{x}, \gamma_{y}\right) \mathbf{U}_{r}(\alpha)$. The fractional angles $\gamma_{x}$ and $\gamma_{y}$ follow from the relations ${ }^{24}$

$$
\begin{gathered}
\exp \left[i\left(\gamma_{x}+\gamma_{y}\right)\right]=\operatorname{det} \mathbf{U}_{\circ}, \\
\cos \left(\gamma_{x}-\gamma_{y}\right)=\operatorname{det} \mathbf{X}_{\circ}+\operatorname{det} \mathbf{Y}_{\circ}
\end{gathered}
$$

where the $\pi$ phase ambiguity can be avoided by choosing $0 \leqslant \gamma_{x}+\gamma_{y}<2 \pi$ and $0 \leqslant \gamma_{x}-\gamma_{y}<\pi$. The rotation angles $\alpha$ and $\beta$ can then be determined from the elements of the matrices $\mathbf{X}_{\circ}$ and $\mathbf{Y}_{\circ}{ }^{24}$ With $\theta_{1}$ and $\theta_{2}$ as the angles that arise in the exponents of the unimodular eigenvalues $\lambda_{n}$ $=\exp \left( \pm i \theta_{n}\right)$ of the unimodular ray transformation matrix $\mathbf{T}_{u}$ [and of the unitary matrix $\mathbf{U}_{\circ}=\mathbf{U}_{r}(\beta) \mathbf{U}_{f}\left(\gamma_{x}, \gamma_{y}\right) \mathbf{U}_{r}(\alpha)$, we easily derive the relationship

$$
\theta_{1,2}=\left(\gamma_{x}+\gamma_{y}\right) / 2 \pm \operatorname{arcos}\left\{\cos (\alpha+\beta) \cos \left[\left(\gamma_{x}-\gamma_{y}\right) / 2\right]\right\}
$$

Hence, unless the rotation angles of the rotators $\mathbf{U}_{r}(\alpha)$ and $\mathbf{U}_{r}(\beta)$ satisfy the condition $\alpha+\beta=0$, the fractional angles $\gamma_{x}$ and $\gamma_{y}$ of the separable fractional Fourier transformer $\mathbf{U}_{f}\left(\gamma_{x}, \gamma_{y}\right)$ are not identical to $\theta_{1}$ and $\theta_{2}$.

While the rotators (with rotation angles $\alpha$ and $\beta$ ) can be realized by simply defining rotated coordinate systems, the separable fractional Fourier transformer (with different fractional angles $\gamma_{x}$ and $\gamma_{y}$ in the two perpendicular directions) can be realized, for instance, by Lohmann's setup (a) as described in Section 2. We then have a combination of two crossed cylindrical lenses (with different focal lengths $f_{x}$ and $f_{y}$ ) preceded and followed by identical distances $z$ of free space, with proper rotations of the coordinate systems before and after the separable fractional Fourier transformer. The $D=2$ fractional angles and the $D(D-1)=2$ rotation angles constitute $D^{2}=4$ degrees of freedom.

The lens in the decomposition of Eq. (57) is described by the symmetric matrix $\mathbf{G}$ (or - $\mathbf{G}$ ), which can be decomposed as

$$
\mathbf{G}=\left[\begin{array}{ll}
g_{11} & g_{12} \\
g_{12} & g_{22}
\end{array}\right]=\mathbf{U}_{r}\left(\varphi_{g}\right)\left[\begin{array}{cc}
g_{1} & 0 \\
0 & g_{2}
\end{array}\right] \mathbf{U}_{r}\left(-\varphi_{g}\right),
$$

where $g_{1,2}=\left(g_{11}+g_{22}\right) / 2 \pm \sqrt{\left[\left(g_{11}-g_{22}\right) / 2\right]^{2}+g_{12}^{2}}$ and $\tan \varphi_{g}$ $=\left(g_{11}-g_{1}\right) / g_{12}=g_{12} /\left(g_{22}-g_{1}\right)=g_{12} /\left(g_{2}-g_{11}\right)=\left(g_{2}-g_{22}\right) / g_{12}$ (see also Ref. 15, Section 10.2.1, Lenses). This decomposition shows a possible realization of the two-dimensional (anamorphic) lens as a combination of two crossed cylindrical lenses whose focal lengths are defined by $g_{1}$ and $g_{2}$, and which is oriented at an angle $\varphi_{g}$. The $D=2$ focal lengths and the $D(D-1) / 2=1$ orientation angle constitute $D(D+1) / 2=3$ degrees of freedom.

The magnifier in the decomposition of Eq. (57) is described by the positive-definite symmetric matrix $\mathbf{S}$ (or $\mathbf{S}^{-1}$ ), which can again be decomposed as

$$
\mathbf{S}=\left[\begin{array}{ll}
s_{11} & s_{12} \\
s_{12} & s_{22}
\end{array}\right]=\mathbf{U}_{r}\left(\varphi_{s}\right)\left[\begin{array}{cc}
s_{1} & 0 \\
0 & s_{2}
\end{array}\right] \mathbf{U}_{r}\left(-\varphi_{s}\right)
$$

[see Eq. (62); see also Ref. 15, Section 10.2.2, Magnifiers]. The (anamorphic) two-dimensional magnifier can thus be realized as a combination of two one-dimensional magnifiers whose magnification factors are defined by $s_{1}$ and $s_{2}$, and which is oriented at an angle $\varphi_{s}$. A one-dimensional magnifier (with reversion) can easily be realized by means of an ideal imaging system built around a thin (cylindrical) lens with focal distance $f$. The input plane of this system is located a distance $z_{i}$ before this lens, whereas its output plane is located a distance $z_{o}$ behind the lens. To get ideal imaging we have of course the condition $1 / z_{i}+1 / z_{o}=1 / f$, and the magnification is then $s$ $=z_{o} / z_{i}$. To compensate for an unwanted phase factor, we need a thin (cylindrical) phase-correcting lens with focal distance $f_{c}$ either in the input plane, with $f_{c}=z_{i}-f$, or in the output plane, with $f_{c}=z_{o}-f$.

Two crossed one-dimensional magnifiers, with different ratios $s_{1}=z_{o, 1} / z_{i, 1}$ and $s_{2}=z_{o, 2} / z_{i, 2}$, but with identical total length $z_{i, 1}+z_{o, 1}=z_{i, 2}+z_{o, 2}$, lead to a two-dimensional, separable magnifier. The $D=2$ magnification factors (and thus the two corresponding focal lengths) and the $D(D-1) / 2$ $=1$ orientation angle constitute again $D(D+1) / 2=3$ degrees of freedom. We remark that the reversion does not disturb us, because it will be compensated by a second reversion that appears in the second magnifier in the decomposition of Eq. (57). Note also that the phasecorrecting lens might be combined with the lens that corresponds to $\mathbf{G}$ (see above).

Of course, instead of using the decomposition of Eq. (57) [or Eq. (58)], the unimodular system $\mathbf{T}_{u}$ can be reduced to the normal Iwasawa decomposition of Eq. (51), like any first-order optical system, and can thus be realized by using less optical elements than described above, in particular two crossed cylindrical lenses for the separable fractional Fourier transformer that arises in the orthosymplectic subsystem, two cylindrical lenses for the magnifier, and two crossed cylindrical lenses for the anamorphic lens (combined with the phase-correcting lenses of the magnifier). In that case, however, the direct relationship between the system parameters and the optical elements is lost.

\section{CONCLUSIONS}

Unimodular systems and their associated signal transformations $\mathcal{R}_{u}$ are perfectly suited for fractionalization. As an example we mention the common Fourier transformation in one dimension; its eigenvalues $\mu_{n}$ [with corresponding eigenfunctions $\left.\Phi_{n}(x)=\mathcal{H}_{n}(x), n=0,1, \ldots\right]$ read $\mu_{n}=\exp (-i n \pi / 2)$ and the eigenvalues $\lambda_{1,2}$ of its associated ray transformation matrix $\mathbf{T}_{f}(\pi / 2)$ read $\lambda_{1,2}=\exp ( \pm i \pi / 2)$. Replacing the angle $\pi / 2$ that arises in the eigenvalues by an arbitrary phase angle leads to one version of the many possible fractionalized forms. This particular fractionalized form, the canonical fractional Fourier transformation, is the one that is commonly used in optics. The same procedure can be applied to all other unimodular transformations.

Now that we have shown that all unimodular systems are similar to a separable fractional Fourier transformer, $\mathbf{T}_{u}=\mathbf{M} \mathbf{T}_{f} \mathbf{M}^{-1}$, we have implicitly shown that the Fourier transformation is the key element in the fractionalization of unimodular canonical integral transformations. Moreover, the cascades of Eqs. (57) and (58) show direct optical realizations of such transformations. 


\section{ACKNOWLEDGMENTS}

The Spanish Ministry of Education and Science is acknowledged for financial support: Ramon y Cajal grant and project TIC 2002-01846 (T. Alieva) and SAB20040018 (M. J. Bastiaans). Stimulating discussions with K. Bernardo Wolf (Universidad Nacional Autónoma de México, Cuernavaca) are gratefully acknowledged.

The authors' e-mail addresses are m.j.bastiaans @tue.nl and talieva@fis.ucm.es.

\section{REFERENCES}

1. A. W. Lohmann, D. Mendlovic, and Z. Zalevsky, "Fractional transformation in optics," in Progress in Optics, Vol. XXXVIII, E. Wolf, ed. (North-Holland, 1998), pp. 263-342.

2. T. Alieva, M. J. Bastiaans, and M. L. Calvo, "Fractional transforms in optical information processing," EURASIP J. Appl. Signal Process. 2005, 1498-1519 (2005).

3. T. Alieva and M. L. Calvo, "Fractionalization of the linear cyclic transforms," J. Opt. Soc. Am. A 17, 2330-2338 (2000).

4. T. Alieva, M. J. Bastiaans, and M. L. Calvo, "Fractional cyclic transforms in optics: theory and applications," in Recent Research and Developments in Optics, S. G. Pandalai, ed. (Research Signpost, 2001), Vol. 1, pp. 105-122.

5. T. Alieva, M. J. Bastiaans, and M. L. Calvo, "Fractionalization of cyclic transformations in optics," in Proceedings of ICOL 2005, the International Conference on Optics and Optoelectronics, Dehradun, India, December 12-15, 2005, Paper IT-OIP-2, pp. 1-15.

6. S. A. Collins, Jr., "Lens-system diffraction integral written in terms of matrix optics," J. Opt. Soc. Am. 60, 1168-1177 (1970).

7. M. Moshinsky and C. Quesne, "Linear canonical transformations and their unitary representations," J. Math. Phys. 12, 1772-1780 (1971).

8. R. K. Luneburg, Mathematical Theory of Optics (University of California Press, 1966).

9. J. W. Goodman, Introduction to Fourier Optics, 2nd ed. (McGraw-Hill, 1996).
10. A. W. Lohmann, "Image rotation, Wigner rotation, and the fractional Fourier transform,” J. Opt. Soc. Am. A 10, 2181-2186 (1993).

11. D. Mendlovic and H. M. Ozaktas, "Fractional Fourier transforms and their optical implementation I," J. Opt. Soc. Am. A 10, 1875-1881 (1993).

12. H. M. Ozaktas and D. Mendlovic, "Fractional Fourier transforms and their optical implementation II," J. Opt. Soc. Am. A 10, 2522-2531 (1993).

13. H. M. Ozaktas, Z. Zalevsky, and M. A. Kutay, The Fractional Fourier Transform with Applications in Optics and Signal Processing (Wiley, 2001).

14. G. A. Korn and T. M. Korn, Mathematical Handbook for Scientists and Engineers: Definitions, Theorems, and Formulas for Reference and Review (McGraw-Hill, 1968).

15. K. B. Wolf, Geometric Optics on Phase Space (Springer, 2004).

16. V. Namias, "The fractional order Fourier transform and its applications to quantum mechanics," J. Inst. Math. Appl. 25, 241-265 (1980).

17. A. C. McBride and F. H. Kerr, "On Namias' fractional Fourier transforms," IMA J. Appl. Math. 39, 159-175 (1987).

18. V. Bargmann, "On a Hilbert space of analytic functions and an associated integral transform, Part I," Commun. Pure Appl. Math. 14, 187-214 (1961).

19. I. E. Segal, "Distributions in Hilbert spaces and canonical systems of operators," Trans. Am. Math. Soc. 88, 12-42 (1958).

20. R. Simon and K. B. Wolf, "Structure of the set of paraxial optical systems," J. Opt. Soc. Am. A 17, 342-355 (2000).

21. M. J. Bastiaans and T. Alieva, "Generating function for Hermite-Gaussian modes propagating through first-order optical systems," J. Phys. A 38, L73-L78 (2005).

22. T. Alieva and M. J. Bastiaans, "Mode mapping in paraxial lossless optics," Opt. Lett. 30, 1461-1463 (2005).

23. R. Simon and N. Mukunda, "Iwasawa decomposition in first-order optics: universal treatment of shape-invariant propagation for coherent and partially coherent beams," J. Opt. Soc. Am. A 15, 2146-2155 (1998).

24. T. Alieva and M. J. Bastiaans, "Alternative representation of the linear canonical integral transform," Opt. Lett. 30, 3302-3304 (2005). 